\title{
On the Spatial Distribution of Stellar Populations in the Large Magellanic Cloud ${ }^{1}$
}

\author{
Jason Harris and Dennis Zaritsky \\ UCO/Lick Observatory and Department of Astronomy and Astrophysics \\ Univ. of California, Santa Cruz, CA, 95064 \\ E-Mail:jharris@ucolick.org, dennis@ucolick.org
}

${ }^{1}$ Lick Bulletin No. 1385

\begin{abstract}
We measure the angular correlation function of stars in a region of the Large Magellanic Cloud (LMC) that spans $2.0^{\circ} \times 1.5^{\circ}$. We find that the correlation functions of stellar populations are represented well by exponential functions of the angular separation for separations between 2 and 40 arcmin (corresponding to $\sim 30 \mathrm{pc}$ and $550 \mathrm{pc}$ for an LMC distance of $50 \mathrm{kpc}$ ). The inner boundary is set by the presence of distinct, highly correlated structures, which are the more familiar stellar clusters, and the outer boundary is set by the observed region's size and the presence of two principal centers of star formation within the region. We also find that the normalization and scale length of the correlation function changes systematically with the mean age of the stellar population. The existence of positive correlation at large separations $(\sim 300 \mathrm{pc})$, even in the youngest population, argues for large-scale hierarchical structure in current star formation. The evolution of the angular correlation toward lower normalizations and longer scale lengths with stellar age argues for the dispersion of stars with time. We show that a simple, stochastic, self-propagating star formation model is qualitatively consistent with this behavior of the correlation function.
\end{abstract}

Subject headings: Magellanic Clouds — stars: evolution — stars:

formation — stars: kinematics 


\section{Introduction}

The evolution of the stellar component of a galaxy can be separated into the star formation history, the internal evolution of those stars, and the dynamical behavior of the system. The study of the star formation histories of local galaxies, in which individual stars are resolved, is increasing rapidly in sophistication (cf. Aparicio et al. 1997, Geha et al. 1998, Tolstoy et al. 1998) and is providing evidence for a wide variety of histories (see Mateo 1999 for a review). Likewise, the theory of stellar evolution is one of the triumphs of modern astrophysics. In contrast, less is known about the initial distribution and subsequent dynamical evolution of stars within these systems. Although most stars form as members of stellar associations (Gomez et al. 1993, Massey et al. 1995), which are themselves distributed in a way that suggests hierarchical structure (Nail \& Shapley 1953, Elmegreen \& Efremov 1996), the quantitative details of the initial stellar distribution and its subsequent evolution remain only weakly constrained on large scales. Stellar associations, which are typically unbound (Blaauw 1964, Kontizas et al. 1994) and loosely defined, will become increasingly dispersed over time and mix with any true field population, making it difficult to associate older stars with a localized star formation event. Even simple issues, such as the relative number of stars that form in and out of associations, remain unresolved.

The detailed study of star forming regions and stellar kinematics has, by necessity, been confined primarily to star forming complexes in our galaxy (cf. Gomez et al. 1993, Larson 1995, Bate et al. 1998, Gomez \& Lada 1998). However, external galaxies may provide a contrasting environment, a viewpoint that is independent of our position within the Milky Way, and an opportunity to examine the behavior of star formation on galactic scales. Galactic-scale star formation patterns have been studied using Cepheids in the Large Magellanic Clouds (Elmegreen \& Efremov 1996), the spatially resolved star formation history in nearby dwarf galaxies (cf. Dohm-Palmer et al. 1997), and the distribution of the most active centers of current star formation in more distant galaxies (cf. Hunter et al. 1998). All of these studies focus on recent star formation (age $\lesssim 5 \times 10^{8}$ years) and therefore provide more information on the distribution of current star formation than on the subsequent dynamical evolution of stellar populations or on any relation that might exist between past and current star formation. Our goal here is to extend the study of star clustering over a wider range of stellar populations, larger areas, and greater lookback times. In particular, we provide a quantitative description of the 
distribution of different stellar populations in the Large Magellanic Cloud (LMC).

The LMC is an ideal laboratory for this study because it contains a variety of stellar populations, is relatively nearby (Madore \& Freedman 1998), has low average dust extinction ( Harris et al. 1997), is close to face-on (inclined by $\sim 30^{\circ}$ to the line of sight, Westerlund 1997), and has a small line-of-sight depth (Caldwell \& Coulson 1986). Any patterns that emerge in the stellar distributions should be useful in constraining both the initial distribution and the dynamical evolution of stellar populations. We do the following with our observations of the stellar populations in the LMC: (1) quantify the clustering properties using the angular correlation function; (2) measure the clustering properties as a function of mean stellar age; (3) identify evidence for hierarchical clustering in the young population over scales smaller than $\sim 550 \mathrm{pc}$; (4) qualitatively test a model of stochastic, self-propagating star formation; (5) describe the possibility of measuring the true field star fraction; and (6) discuss the impact of dynamical evolution on studies of the stellar populations.

\section{Data and Analysis}

The data used in this study come from our $U B V I$ photometric survey of the Large Magellanic Cloud (LMC) and the Small Magellanic Cloud (SMC). Zaritsky et al. (1997) present a description of the survey. Our final catalog will consist of astrometry ( $\sim 0.5$ arcsec positional rms error) and photometry $\left(\sigma_{V}=0.09 \mathrm{mag}\right.$ at $\left.V=20 \mathrm{mag}\right)$ for $\sim 25$ million stars. In this paper, we examine the section of the LMC discussed by Zaritsky et al. , which is centered at $\left(5.2^{h},-67^{\circ} .4\right)$ and is approximately $2.0^{\circ} \times 1.5^{\circ}$ in extent. The $B-V, V$ Hess diagram for this region of the LMC is shown in Figure 11.

\subsection{The Stellar Angular Correlation Function}

A common and natural measurement of the distribution of objects on the sky is the angular correlation function. The evolution of the stellar spatial distribution can be quantified by examining the behavior of the angular correlation function for stellar populations of different ages. We define the angular correlation function, $\omega(\theta)$, for a given stellar population to be

$$
\omega(\theta) \equiv \frac{f(\theta)}{f_{R}(\theta)}-1,
$$


where $f(\theta)$ is the auto-correlation function of the stellar population, and $f_{R}(\theta)$ is the cross-correlation function of the stellar population with a randomly distributed population. Isolating field stellar populations of a single age in a complex system like the LMC is nearly impossible (see Elmegreen \& Efremov 1996 for one exception) and so we rely on isolating populations of differing mean ages. Assuming that star formation in this region occurred over at least the past 2 Gyr and had an extended duration (Bertelli et al. 1992, Holtzman et al. 1997, Geha et al. 1998), we separate populations that are sequential in mean age by sorting the upper main sequence (MS) stars by magnitude. We define upper MS stars to lie in the region of the CMD bounded by $20.5-e^{(6.6-12(B-V))}<V<20$ and distribute these stars into eight main sequence bins (referred to as MS1 through MS8). The peculiar definition of the lower $V$ magnitude cut was set interactively to crudely differentiate MS stars from evolved stars ( $c f$. Figure 1). The eight MS bins are defined in Table 1 and labeled in Figure 1. MS1 and MS2 span a larger range of $V$ magnitudes because there are fewer stars per magnitude at these luminosities.

Clustering among the youngest stars (MS1) is evident in Figure 2. The quantitative measure of the clustering is plotted in Figure 3 in log-linear and $\log$ - $\log$ coordinates to illustrate what we find to be a general trend at these separations: $\omega(\theta)$ is better represented by an exponential function than by a power law. The best-fit exponential and power law for separations $2<\theta<18$ arcmin, which corresponds to projected physical separations of 30 to 260 pc (adopting $D_{L M C}=50 \mathrm{kpc}$, Madore \& Freedman 1998), are plotted for comparison. The reduced $\chi^{2}$ is 1.5 for the exponential fit and 35.9 for the power law fit. This result is somewhat surprising since clustering of astronomical objects is often well-described by power law correlation functions (as in various studies of Galactic star forming regions, cf. Gomez et al. 1993, Larson 1995, Nakajima et al. 1998). However, our result is not in conflict with the studies of Galactic associations because those measure the stellar correlation function on much smaller physical scales (typically less than 1 pc). For $\theta<2 \operatorname{arcmin}(30$ pc), $\omega(\theta)$ deviates sharply from the exponential fit because of the presence of tight stellar groupings, the stellar clusters, and may be better represented by a power-law. At large separations, $\omega(\theta)$ again deviates from the fitting function, but $\omega(\theta)$ at these separations is affected by the cross-correlation between the two large stellar aggregates in this region ( $c f$. Figure 2) and by the limited size of the observed region.

To determine the smallest angular separation at which the finite size of the region begins to significantly degrade the results, we calculate $\omega(\theta)$ for red 
clump stars $(0.70<B-V<1.2 \mathrm{mag}$ and $18.8<V<19.6 \mathrm{mag}$ (see Figure (1)) in three concentric subregions of sizes $1.45^{\circ} \times 1.45^{\circ}, 1.0^{\circ} \times 1.0^{\circ}$ and $0.5^{\circ} \times 0.5^{\circ}$. We choose these stars for the test because they are distributed nearly uniformly throughout the region. Two qualitative oddities are evident in the measured correlation functions (Figure 8). First, the $\omega(\theta)$ 's are systematically offset from each other because there is a surface density gradient in the red clump (RC) population. The smallest subregion has the least contrast between the highest and lowest surface density, so the correlation values are depressed. Second, all three correlation functions have a steep drop at angular separations of 0.5 to 0.65 times the size of the region. This drop occurs because of the limited size of the region and the mathematical construction of the correlation function such that there is zero correlation at large separations. We conclude that if we restrict our study to angular separations that are less than half the smaller dimension of the observed region (corresponding to $\theta<40$ arcmin for our full region), then edge effects will not affect the shape of the correlation function, even though the global normalization is undetermined.

The evolution of clustering is evident in the comparison of $\omega(\theta)$ for the eight MS populations (Figure 4). We calculate least-squares fits to each correlation function over the angular separation range $2<\theta<16$ arcmin for MS1, MS2 and MS3, and $2<\theta<20$ arcmin for MS4 through MS8. The $\chi^{2}$ values for each fit are $\lesssim 1$, indicating that the scatter about the exponential is consistent with the internal uncertainties, and the fits appear to extrapolate well to larger separations that are $<40$ arcmin. The correlation function scale length increases and its normalization (which we define as the extrapolation of the best-fit exponential to zero separation) decreases as the mean luminosity of stars decreases (Table 1 and Figure 5). Because mean age increases as MS bin magnitude increases, we suggest that the behavior of the correlation function is the result of the dispersal of stellar populations as they age (alternatively, the pattern of star formation may change with time in a way to produce similar behavior).

To determine whether the correlation function continues to weaken in even older populations, we calculate $\omega(\theta)$ for some of the oldest stars that we can identify from our data, the red clump population. The exact age distribution of the RC stars is dependent on the poorly known star formation history, but the mean age is at least a few Gyr (for the star formation history derived by Holtzman et al. 1997, 73\% of the RC stars are older than 2 Gyr; Cole 1998) and therefore significantly older than the MS populations discussed above. The spatial distribution of RC stars is shown in Figure 6 and the corresponding 
$\omega(\theta)$ is shown in Figure [7. The reduced $\chi^{2}$ for the exponential fit is 0.47 and 6.6 for the power law. The trend of a decreasing normalization and a larger scale length with increasing age continues (see Fig. 5). Finally, the decline in the correlation function at small radii for the $\mathrm{RC}$ stars is most likely an artifact arising from the greater incompleteness for fainter stars in the dense cluster cores.

\section{Discussion}

We have demonstrated that $\omega(\theta)$ for this region of the LMC is well represented by an exponential function and that it changes systematically with the mean age of the stellar population. What do these observations imply about the evolution of the spatial distribution of stars in the LMC? Assuming that the clustering properties of newly formed stars have remained constant over the past billion years, the systematic change in $\omega(\theta)$ implies that dynamical evolution results in the dispersion of initially highly clustered stars. The relatively weaker, but nonzero, $\omega(\theta)$ of the RC population suggests that some clustering signatures may be detectable for at least a few Gyr. The quantitative measure

presented here enables us to directly tests models that attempt to explain these observations.

In this section, we present highly simplified models that grossly reproduce the observed behavior. We begin by examining the correlation function from a stellar population with an exponential surface density gradient. We find that such a gradient does not precisely reproduce the observed correlation among RC stars, a population that is expected to be well-mixed. Then we examine simple clustering geometries to understand the origin of the observed exponential correlation function. We extend this analysis by constructing a model of self-propagating star formation plus simple dynamical evolution that reproduces the observed systematic trends in $\omega(\theta)$ with stellar age. While this model is not unique and highly simplified (e.g. it does not include self-gravity or hydrodynamics), it demonstrates that given plausible assumptions, the observed clustering properties can be straightforwardly reproduced. Given the qualitative agreement between our extremely naive model and the observations, we assert that more sophisticated models based on the same geneal principles will be able to quantitatively match the observed behavior. Finally, we discuss some implications of our findings on studies of the field star formation fraction, star formation histories, and stellar dynamics. 


\subsection{A Non-Uniform Underlying Population}

To test whether an underlying stellar density gradient can reproduce the observed correlation function of our most mixed population (the RC stars), we simulate a stellar population with an exponential surface density gradient. We center the artificial population coincident with the optical center of the LMC $\left(5.33^{h},-69^{\circ} .46\right.$; de Vaucouleurs \& Freeman 1972), which is about $2^{\circ}$ south of the observed region, and adopt a radial length scale of 101 arcmin (Bothun \& Thompson (1988)). The resulting $\omega(\theta)$ of stars placed randomly with this exponential distribution, and only within a rectangular boundary corresponding to the observed region has a significantly greater normalization than that observed (Figure 9). Bothun \& Thompson's radial scale length is based on $B$-band surface photometry, and so it is possible that the older, redder RC population has a different radial exponential profile. Using radial length scales of 115 arcmin and 130 arcmin, we find that we reduce the normalization of the correlation function sufficiently but still cannot recover the observed shape, in particular the rise at smaller separations ( $\theta \lesssim 16$ arcmin). We conclude that an exponential gradient in the surface density of RC stars is insufficient to entirely explain the observed $\omega(\theta)$. The residual structure in the distribution of $\mathrm{RC}$ stars might be the result of either an RC population that is still dynamically evolving toward a uniform exponential disk distribution, or of large coherent structures in the LMC (e.g. spiral arms). This ambiguity should be resolved once a larger section of the LMC has been cataloged.

\subsection{The Exponential Correlation Function}

The observed $\omega(\theta)$ 's are well-fit by exponential functions over a large range of angular separations. To determine whether exponential correlation functions can be generated by extremely simple stellar distributions of stars, we calculate $\omega(\theta)$ for several representative artificial distributions. First, we simulate single stellar clusters with Gaussian and exponential radial density profiles embedded in a uniform stellar background. Each simulation contains 6000 stars (a compromise between spatial resolution and computational time), half of which are placed according to the cluster profile and half of which are distributed uniformly across the region. We assign characteristic radial scales ( $\sigma$ for Gaussians, scale length for exponentials) of 2.5\%, 5\%, $10 \%$ and $25 \%$ of the simulated region's size, to fully explore the dependence of the correlation function on cluster size while avoiding edge effects (see $\$ 2.1$ ).

The results from these simulations are shown in Figure 10. The Gaussian 
and exponential clusters produce $\omega(\theta)$ 's that are virtually indistinguishable and consistent with exponential correlation functions. Decreasing the characteristic length scale steepens the correlation function, increases the normalization, and decreases $\theta_{\max }$, the angular separation at which the stars become uncorrelated $\left(\theta_{\max }\right.$ is a few times the characteristic length scale in each case). Because observed stellar clusters in the LMC are typically an arcminute or less in diameter (Hodge 1988), the observed positive correlations on scales larger than a few arcmin must arise from larger single structures or correlations among clusters.

We demonstrate that random distributions of clusters cannot generate the observed correlation amplitudes at large $\theta$ by simulating 20 randomly placed star clusters, each with an exponential radial profile and a radial scale length of 1 arcmin. The resulting $\omega(\theta)$ 's from four realizations of this simulation (Figure 11) are highly irregular and fail to reproduce the observations. A more complex model, with correlated cluster positions, is required.

\subsection{The Stochastic Self-Propagating Star Formation Model}

The presence of large scale structure among the young stellar populations of the LMC is well established (Nail \& Shapley 1953, Hodge \& Lucke 1970, Elmegreen \& Efremov 1996). To investigate whether the simple evolution of such clustering can reproduce the temporal behavior of the observed large-scale exponential correlation functions, we use a simple model of stochastic, self-propagating star formation (SSPSF) to construct artificial distributions of hierarchically clustered stars. Traditionally, SSPSF is used to explain the structure of individual star forming regions (Dopita et al. 1985, Feitzinger et al. 1987, Elmegreen \& Efremov 1996), but we employ it here to account for the observed structure of a mixed population in the field of the LMC. We allow the stellar distributions to evolve over time (solely as a result of random velocities) and test whether such a model reproduces the observed trend in clustering properties with stellar population age.

Our SSPSF model contains both a field and a cluster mode of star formation. In both modes, the formation of individual stars is stochastic. Correlation among clusters will arise if cluster formation depends on the previous generation of star formation. There are various possible physical mechanisms that may produce correlated cluster formation: stellar winds (Scalo \& Chappell 1999), a pre-existing hierarchical structure in the interstellar medium (Larson 1995), gamma-ray bursts (Efremov et al. 1998), and supernovae explosions 
(Tenorio-Tagle \& Bodenheimer 1988). In our models, we employ the supernova hypothesis, but any mechanism that results in correlated clusters over the physical and temporal scales discussed here will produce similar results. We trigger cluster formation with supernovae that occur in nearby clusters. Our justification for allowing only cluster supernovae to trigger cluster formation is that field supernovae may not exist in environments sufficiently dense to form clusters (when field supernovae are allowed to trigger cluster formation in the models, the simulated $\omega(\theta)$ is weaker than observed). The goal of our modeling is not to derive the physical parameters for the SSPSF process; rather it is to demonstrate that a fairly straightforward model grounded in processes that are generally believed to occur in galaxies (dynamical relaxation of stars, correlated star formation, and self-propagation of star formation) can lead to clustering properties that are similar to those observed. More specific inferences will require measurement of the correlation function across the entire LMC and dynamically self-consistent models that have a more detailed, physically motivated prescription for star formation (see Scalo \& Chappell 1999 for one such example).

The implementation of our model is straightforward. Stars are formed at a constant rate, and each star is assigned an initial position, an initial velocity, and a mass. A fraction $f$ of stars are formed in the field, the remainder in clusters. Field star positions are drawn randomly from an exponential gradient surface density distribution that matches that observed for the LMC ( $c f$. Bothun \& Thompson 1988). Field stars are assigned zero velocity, in order to preserve the surface density gradient of their initial distribution (in a more realistic model, the gradient would be preserved because the stellar distribution is in dynamical equilibrium). Cluster stars are distributed with an exponential radial profile of characteristic angular scale $\Delta \theta$, centered on one of the simulation's currently active clusters. Cluster stars have a Gaussian, isotropic velocity distribution with mean zero and a one-dimensional velocity dispersion $\sigma_{v}$. The stellar mass is drawn from a Salpeter initial mass function (IMF) between $2.26 M_{\odot}$ and $100 M_{\odot}$. The lower mass limit corresponds approximately to the faintest stars we study here $(V=20 \mathrm{mag})$, and the upper mass limit corresponds approximately to the most massive stars observed in the LMC (Massey et al. 1995, Massey \& Hunter 1998).

The stars evolve kinematically in the simplest possible manner. Each star moves according to its initial assigned velocity until it has reached the end of its main sequence lifetime, at which time the star is removed from the simulation. The main sequence lifetime is determined from a quadratic fit to the $\log \left(\tau_{M S}\right)$ vs. 
$\log (M)$ data tabulated in Fagotto et al. (1994). Stars that reach the boundary of the simulated region are reflected back into the region (this is effectively a periodic boundary condition that maintains a constant local density). There are no gravitational interactions in the simulation. Because OB associations typically have mass densities significantly below that at which clusters become unbound ( $\rho \lesssim 0.1 \frac{M_{\odot}}{p c^{3}}, \overline{\text { Bok 1934) }}$, the stars are not strongly affected by the association's self gravity, especially at the angular separations ( $>2$ arcmin) that we are examining. Finally, we do not include the effect of differential rotation in our simulation because the H I rotation curve of Kim et al. (1998) indicates that our field is within the solid-body rotation region. Initial models with differential rotation quickly erased any initial correlations. Our survey will eventually include regions of the LMC for which differential rotation should be important.

Propagating star formation is implemented through cluster formation that is triggered by nearby supernovae. If a cluster star has $M>8 M_{\odot}$, then that star becomes a supernova at the end of its lifetime and it may trigger, with probability $P_{t}$, the formation of a new cluster within a spherical shell of inner radius $\theta_{t}$ and thickness $\delta \theta_{t}$, centered on the location of the supernova. Each viable cluster center exists for a finite time, $t_{c l}$, after which it ceases to be a site of localized star formation. The number of active clusters is self-regulated by having $P_{t}$ decay exponentially with the current number of active clusters.

The physical parameters as described here are summarized in Table Q 2 , with some constraints from the literature, where available. The parameters that are not constrained by observations are adjusted so that the simulated $\omega(\theta)$ 's reasonably match those observed, although we have not completely explored all of parameter space. The simulation is allowed to run for $3 \mathrm{Gyr}$, which encompasses two generations of the longest-lived stars in the MS8 bin $\left(2.26 M_{\odot}\right.$ corresponds to a main sequence lifetime of $1.3 \mathrm{Gyr}$ ). After $3 \mathrm{Gyr}$, the current projected positions and the masses of all surviving stars are examined. The simulated data are analyzed in the same manner as the observations.

The model provides $\omega(\theta)$ 's that grossly match the observations. The eight MS correlation functions from one realization of our best model ( $c f$. Table 2) are shown in Figure 12. We ran eight realizations of this model, varying only the random seed. Each realization resulted in a unique sequence of best-fit scale lengths and normalizations, but the general trend is indicated in Figure 13, in which we plot the mean values of the fit parameters from our eight realizations. The errorbars indicate the standard deviation of the eight realizations about the mean values. Also plotted is the sequence of observed scale lengths and normalizations from Figure 5. Although the observed sequence of $\omega(\theta)$ 's is 
not quantitatively consistent with this model, we have reproduced the two fundamental characteristics of the observed $\omega(\theta)$ 's : (1) the simulated $\omega(\theta)$ 's are exponential over a large range of angular separations, and (2) the simulated $\omega(\theta)$ 's decrease in normalization and increase in scale length for stars drawn from fainter main sequence bins.

More realistic models will need to include: (1) gravitational interactions among the stars and clusters/associations, (2) a global galactic gravitational potential, (3) a more realistic star formation history (cf. Alcock et al. 1999), (4) a detailed distribution of the interstellar medium and a prescription for how it determines local star formation, and (5) a more detailed and realistic treatment of the self-propagation of star formation.

\subsection{Implications}

The current spatial distribution of stars in the LMC is the result of a mixture of dynamically relaxing and hierarchically clustered stellar populations of various ages. The distribution is suggestive of an SSPSF process, although there are alternate ways to produce hierarchical clustering of stars (e.g., Elmegreen \& Efremov 1996, Scalo \& Chappell 1999). Our model is sufficiently limited that we have avoided drawing any but the most general conclusions from it; rather we use the model to demonstrate one way in which the observed structures may arise and to examine how such a model may answer additional questions. Next we describe how one might determine the field/cluster star formation fraction, a quantity that is otherwise very difficult to measure, and illustrate the limitations of star formation studies over areas of limited physical scale.

\subsubsection{The Field Star Fraction}

The fraction of stars that form in isolation rather than in clusters and associations is poorly constrained. The field star formation fraction in the LMC is claimed to be nonzero because of the existence of isolated, massive stars. At their expected velocities of $\sim 3 \mathrm{~km} \mathrm{~s}^{-1}$, these stars cannot have traveled from the nearest cluster or association to their present location during their lifetime (Massey et al. 1995). Massey et al. also found that the logarithmic slope of the initial mass function is much steeper for this field component $(\Gamma=-4.1 \pm 0.2)$ than for stars in associations and clusters $(\Gamma=-1.3 \pm 0.3)$. This difference in IMF slopes has tremendous implications for our understanding of galaxy evolution and star formation, and so it is critical to determine the relative 
numbers of field and cluster stars. Unfortunately, determining whether stars formed in the field or in associations becomes intractable on a case-by-case basis for all but the shortest-lived stars.

Models of the stellar correlation function enable a statistical approach to the problem. In our models, the best match to the observed spatial distribution of stars is achieved when the field star formation fraction, $f$, is 0.75 . However, by adjusting parameters we are also able to find an acceptable match with $f=0.5$. Despite the non-uniqueness of the model, we do find that some field star formation is necessary to balance the strong correlation of the clusters. This result may indicate that there is field star formation or that actual LMC clusters are larger and more irregular than in our simulations. One can use similar models to determine how large, or irregular, associations need to be, or how fast stars need to move, in order to find models with $f=0$ acceptable. Although our models are too preliminary to provide a reliable measurement of $f$, we propose that more sophisticated models could utilize $\omega(\theta)$ to constrain $f$.

\subsubsection{Limitations on the Study of the Star Formation History}

The dynamical evolution of stellar populations limits our ability to recover the star formation histories of local galaxies, both temporally and spatially. To illustrate, suppose that one observes a field of angular extent $\theta$ centered on a cluster at a distance $D$ in which a burst of star formation of duration $\delta \tau$ just ended. If stars are dispersing with a typical velocity $v \mathrm{~km} \mathrm{~s}^{-1}$, then these stars can propagate from the center of the cluster to the edge of the observed region in time $t$ :

$$
t(M y r)=0.98\left(\frac{D}{\mathrm{pc}}\right)\left(\frac{\mathrm{km} / \mathrm{s}}{v}\right) \sin (\theta / 2) .
$$

If the observed cluster is an OB association in the LMC $(D=50 \mathrm{kpc})$, with typical values $v=3 \mathrm{~km} \mathrm{~s}^{-1}$ and $\theta=2.4$ arcmin (HST WFPC2 field), the center-to-edge diffusion time is only $6 \mathrm{Myr}$. Therefore, stars older than $6 \mathrm{Myr}$ may have dispersed beyond the observed region and the relative fraction of stars older than 6 Myr is underestimated. This bias increases with increasing stellar age. Without accounting for this effect, one would underestimate the mean age of the cluster, distort the inferred star formation rate, and infer either mass segregation or the inward propagation of star formation.

The spatial resolution of the star formation histories is also affected. A stellar population of age $\tau$ has typically diffused away from its formation site by 
an angle $\theta$ :

$$
\theta(\tau)=1.03\left(\frac{v}{\mathrm{~km} / \mathrm{s}}\right)\left(\frac{\tau}{\mathrm{Myr}}\right)\left(\frac{\mathrm{pc}}{D}\right),
$$

for small $\theta$. In the LMC (again assuming a typical $v=3 \mathrm{~km} \mathrm{~s}^{-1}$ and $D_{L M C}=50$ $\mathrm{kpc}), \theta=0.2 \tau$ arcmin. Resolved spatial structure in the star formation history on angular scales smaller than $\theta(\tau)$ is either spurious, or the result of a structure that is gravitationally maintained (either bound or sufficiently decelerated by self gravity). In such a model, our ability to resolve spatial structure in the star formation history is a linear function of the age of the population.

Although these observational biases have been appreciated, the observations presented here demonstrate that population segregation exists out to at least nearly a degree and for stars down to $\mathrm{V}=20$ in the LMC. Therefore, all but the studies of the oldest stars (which one expects to be well mixed) will be affected.

\section{Summary}

We examine the spatial distribution of stars in a region of the LMC and find evidence for the hierarchical clustering and dynamical relaxation of stellar populations. We observe (1) that the stellar angular correlation functions of different stellar populations are exponential over separations up to 40 arcmin (the upper limit on the angular scale of our correlation measurement is set by the current size of the observed region), and (2) that the parameters of the angular correlation function depend on the mean stellar age of the population (higher normalization and smaller scale length for younger populations).

We proceed to interpret these observations through various simple models. We argue that correlation functions that are exponential over separations many times greater than the characteristic cluster size require correlated cluster positions. We demonstrate that hierarchical cluster distributions and the evolution of the correlation function are consistent with a model of stochastic self-propagating star formation and the dispersion of stars away from the sites of their formation with time. The model has numerous assumptions and free parameters, so we do not focus on quantitative results from this modeling. From the qualitative success of the model, we conclude that the observed behavior is not unexpected and that our observations provide detailed quantitative constraints for more sophisticated models.

We discuss how these observations may be used to determine the fraction of stars that formed in the field rather than in clusters or associations. Because it 
has been suggested that the field IMF is significantly different than the cluster IMF (Massey et al. 1995), the relative number of stars in the two populations is critical to reconstructing the star formation history of the LMC, and presumably of any other galaxy. Although our current models are too preliminary to resolve this issue, more sophisticated models may be able to utilize the angular correlation functions of stellar populations to reliably address this issue.

We describe how the observed evolution of the stellar spatial distribution can affect studies of the star formation history. By not accounting properly for the dispersal of stars from the sites of star formation, one can underestimate the mean age of the stars formed from a current site of star formation, distort the inferred star formation history, and incorrectly infer either mass segregation or the propagation of star formation.

In conclusion, the systematic behavior of the angular correlation functions quantifies what is visually apparent - that star formation in the LMC is highly clustered and that older stars are more diffusely distributed. Nail and Shapely (1953) drew attention to this fact over 40 years ago, but by quantifying the clustering we can begin to address additional issues such as the field star fraction, the importance of propagating star formation, and the effect of the dispersal of stars with age on other measurements. By combining the angular correlation function with radial velocity measurements we will be able determine the age-velocity dispersion relation in a galaxy other than our own. Eventually, the Magellanic Clouds may provide less ambiguous, more powerful data than that obtained in our galaxy with which to understand the dynamical evolution of stellar populations within galaxies.

Acknowledgments: JH would like to thank Eva Grebel, for her valuable discussions of issues pertaining to the dynamical evolution of star clusters. DZ acknowledges financial support from an NSF grant AST-9819576, a NASA LTSA grant (NAG-5-3501), fellowships from the David and Lucile Packard Foundation and the Alfred P. Sloan Foundation, and from the Dudley Observatory for the construction of the Great Circle Camera. 


\section{REFERENCES}

Alcock, C. et al. (The MACHO Collaboration) 1999, AJ, in press (astroph/9811240)

Aparicio, A., Gallart, C., \& Bertelli, G. 1997, AJ, 114, 680

Bate, M. R., Clarke, C. J., McCaughrean, M. J. 1998, MNRAS, 297, 1163

Bertelli, G., Mateo, M., Chiosi, C., Bressan, A. 1992, ApJ, 388400

Blaauw, A. 1964, ARA\&A, 2, 213

Bok, B. J., 1934, Harvard Circ., 384

Bothun, G. D. \& Thompson, I. B. 1988, AJ, 96, 877

Caldwell, J. A. R. \& Coulson, I. M. 1986, MNRAS, 218, 223

Cole, A. A. 1998, ApJ, 500137

de Vaucouleurs, G. \& Freeman, K. C. 1972, Vistas in Astronomy, 14, 163

Dohm-Palmer, R.C. et al. 1997, AJ, 114, 252

Dopita, M. A., Mathewson, D. S., \& Ford, V. L. 1985, ApJ, 297, 599

Efremov, Y. N., Elmegreen, B. G., \& Hodge, P. W. 1998, ApJ, 501, 163

Elmegreen, B.G. \& Efremov, Y. N. 1996, ApJ, 466, 802

Fagotto, F., Bressan, A., Bertelli, G., \& Chiosi, C. 1994, A\&AS, 105, 29

Feitzenger, J. V., Haynes, R. F., Klein, U., Wielebinski, R., \& Perschke, M. 1987, Vistas in Astronomy, 30, 243

Geha, M. C., et al. 1998, AJ, 115, 1045

Girardi, L., Chiosi, C., Bertelli, G., \& Bressan, A. 1995, A\&A, 293, 87

Gomez, M., Hartmann, L., Kenyon, S. J., \& Hewett, R. 1993, AJ, 105, 1927

Gomez, M. \& Lada, C. J. 1998, AJ, 115, 1524

Harris, J., Zaritsky, D., \& Thompson, I. 1997, AJ, 114, 1933

Hodge, P. W. 1988, PASP, 100, 1051

Hodge, P. W. \& Lucke, P. B. 1970, AJ, 75, 933

Holtzman, J. A. 1997, AJ, 113, 656

Hunter, D. A., Elmegreen, B. G., \& Baker, A. 1998, ApJ, 493, 595

Kim, S., Staveley-Smith, L., Dopita, M.A., Freeman, K.C., Sault, R. J., Kesteven, M. J., \& McConnell, D. 1998, ApJ, 503, 674 
Kontizas, M., Kontizas, E., Dapergolas, A., Argyropoulos, S., \& Bellas-Velidis, Y. 1994, A\&AS, 107, 77

Larson, R. B. 1995, MNRAS, 272, 213

Lupton, R. H., Fall, S. M., Freeman, K. C., Elson, R. A. W. 1989, ApJ, 347, 201

Madore, B. F. \& Freedman, W. L. 1998, ApJ, 492, 110

Mateo, M. 1999, ARA\&A, in press

Massey, P. \& Hunter, D. A. 1998, ApJ, 493, 180

Massey, P., Lang, C. C., DeGioia-Eastwood, K., \& Garmany, C. D. 1995, ApJ, 438,188

Nail, V. McK \& Shapley, H. 1953, Proc. Nat. Acad. Sci., 39, 358

Nakajima, Y., Tachihara, K., Hanawa, T., \& Nakano, M. 1998, ApJ, 497, 721

Scalo, J., \& Chappell, D. 1999, ApJ, 510, 258

Tenorio-Tagle, G. \& Bodenheimer, P. 1998, ARA\&A, 26, 145

Tolstoy, E. et al. 1998, AJ, 116, 1244

Westerlund, B.E. 1997, The Magellanic Clouds, Cambridge Astrophys. Series 29 (Cambridge, New York: Cambridge University Press)

Wood, P. R., Bessell, M. S., \& Paltoglou, G. 1985, ApJ, 290, 477

Zaritsky, D., Harris, J., \& Thompson, I. 1997, AJ, 114, 1002 
Table 1. Main Sequence Bins

\begin{tabular}{ccccc}
\hline \hline Bin & $V$ range $[\mathrm{mag}]$ & Mass range $\left[M_{\odot}\right]$ & Scale Length $[\operatorname{arcmin}]$ & Normalization \\
\hline 1 & $10.0-16.0$ & $30.0-11.9$ & 4.5 & 1.12 \\
2 & $16.0-17.0$ & $11.9-8.1$ & 4.7 & 0.26 \\
3 & $17.0-17.5$ & $8.1-6.5$ & 5.4 & -0.59 \\
4 & $17.5-18.0$ & $6.5-5.3$ & 7.7 & -1.24 \\
5 & $18.0-18.5$ & $5.3-4.3$ & 9.1 & -1.51 \\
6 & $18.5-19.0$ & $4.3-3.4$ & 11.6 & -1.82 \\
7 & $19.0-19.5$ & $3.4-2.6$ & 13.2 & -2.07 \\
8 & $19.5-20.0$ & $2.6-2.3$ & 16.8 & -2.19 \\
\hline
\end{tabular}

Table 2. SSPSF Model Parameters

\begin{tabular}{lrrll}
\hline \hline Parameter & \multicolumn{1}{c}{ Description } & Value & Constraint & Reference \\
\hline$f$ & fraction of stars in field mode & 0.75 & $>0$ & Massey et al. (1995) \\
$n_{c}$ & Number of clusters at which $p_{t}$ drops to 0.5 & 0.6 & & \\
$\sigma_{v}$ & line-of-sight velocity dispersion & $2.75 \mathrm{~km} \mathrm{~s}^{-1}$ & $\lesssim 3 \mathrm{~km} \mathrm{~s}^{-1}$ & Lupton et al. (1989) \\
$\Delta \theta$ & angular size of active star clusters & 2.3 arcmin & 0.3 arcmin & Hodge (1988) \\
$\theta_{t}$ & inner radius of SN trigger annulus & 0.6 arcmin & & \\
$\delta \theta_{t}$ & thickness of SN trigger annulus & 0.6 arcmin & & \\
$t_{c l}$ & star forming lifetime of clusters & $1 \mathrm{Myr}$ & $\sim 5 \mathrm{Myr}$ & Elmegreen \& Efremov 1996 \\
\hline
\end{tabular}




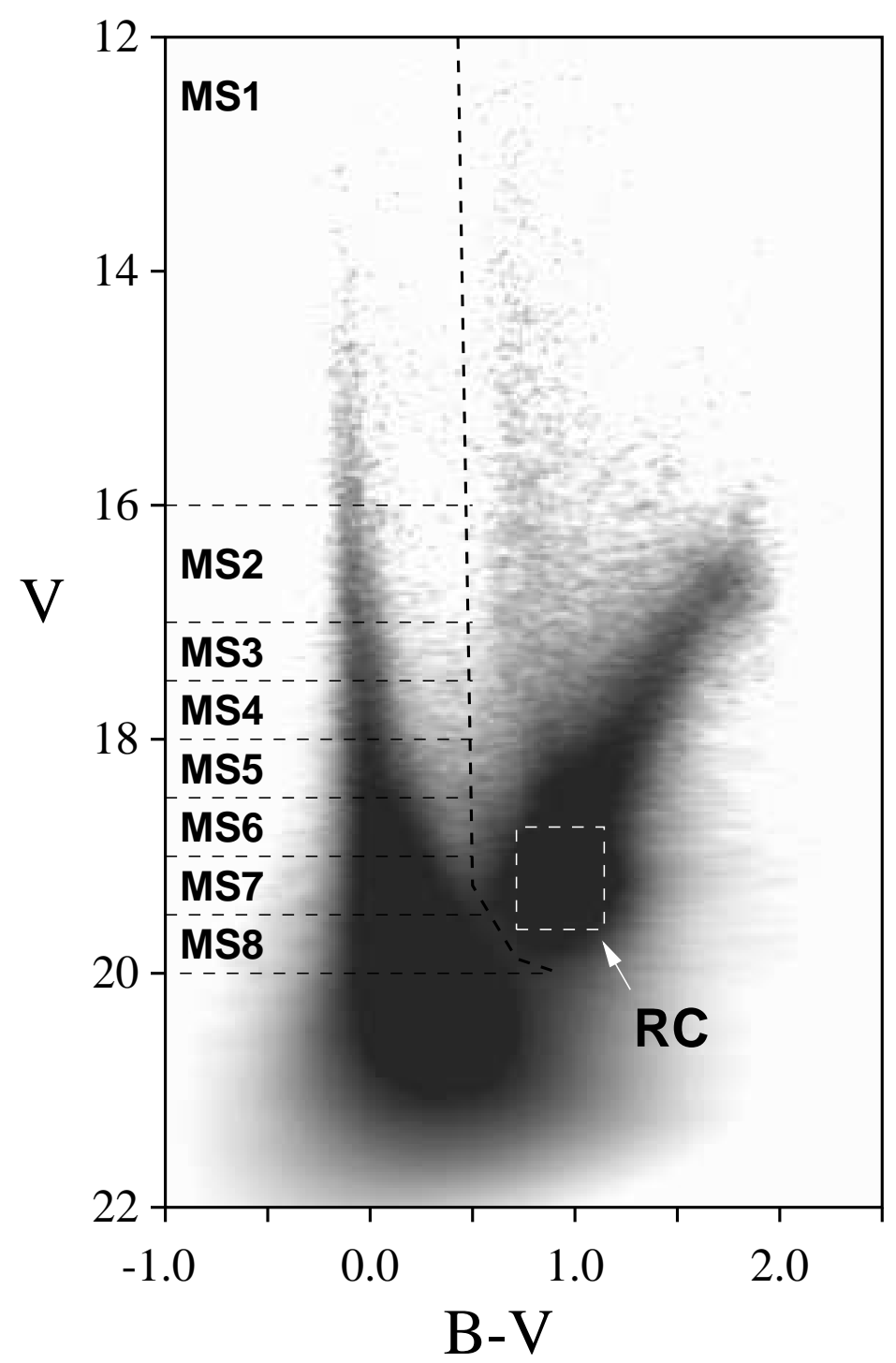

Fig. 1.- A $B-V, V$ Hess diagram for our LMC drift scan survey image. Over 1.1 million stars are included. Each star is represented by a two-dimensional Gaussian with dimensions given by the observational uncertainties. Main sequence stars and red clump stars have been photometrically isolated as shown. 


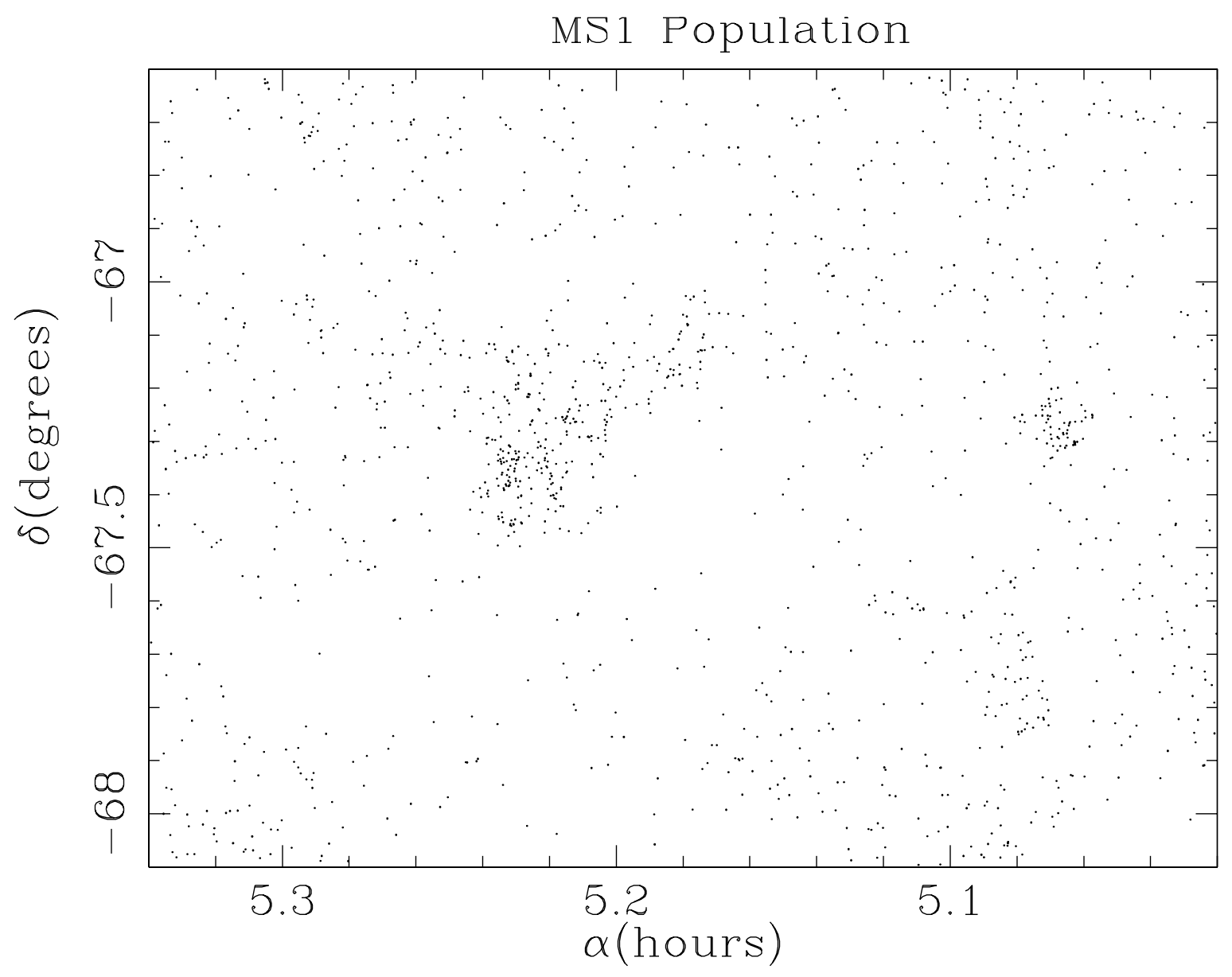

Fig. 2.- The distribution on the sky of the MS1 population, main sequence stars with $V<16$ mag (see Table 11). 

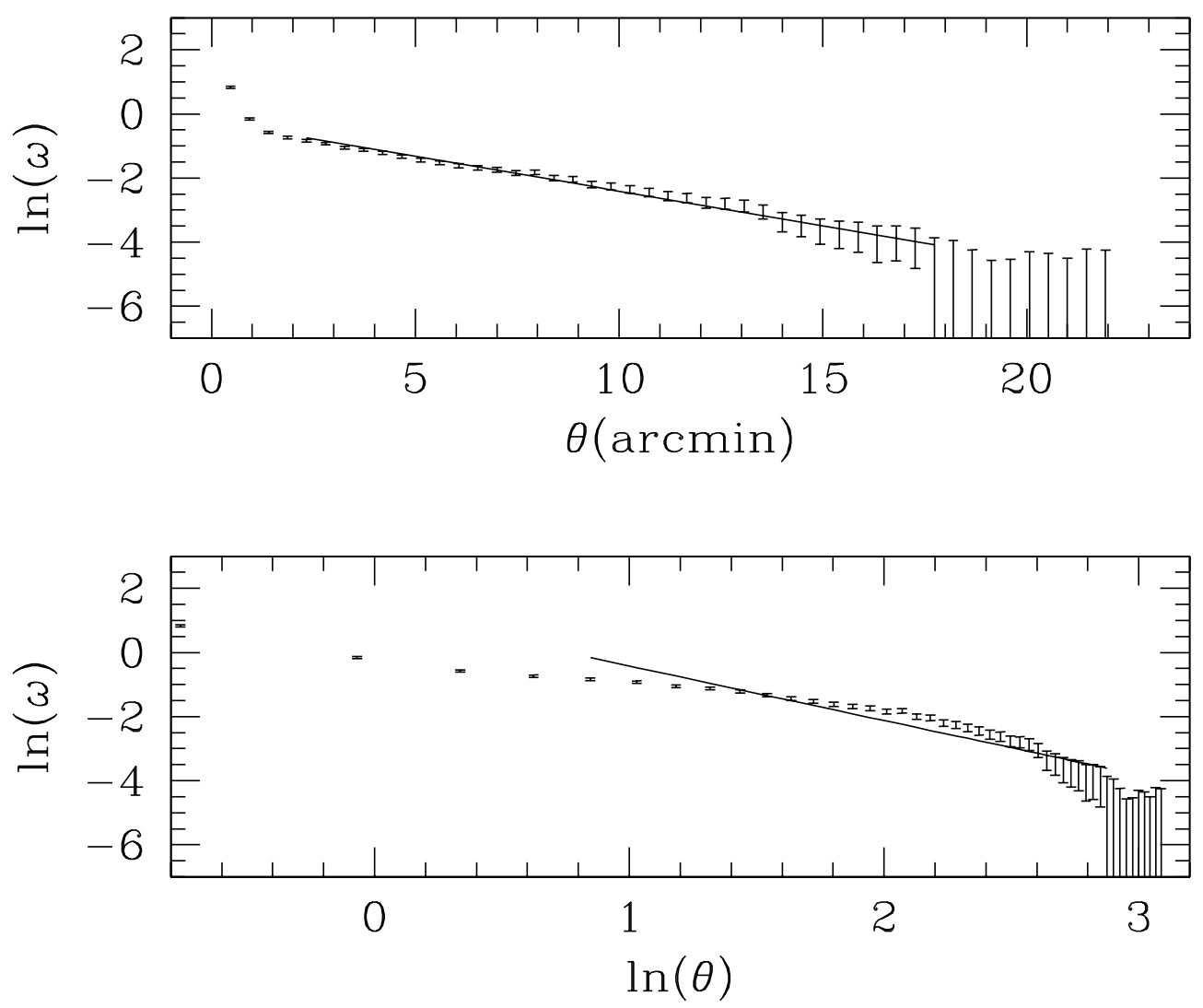

Fig. 3. - The correlation function, $\omega$, of the MS1 population. Upper panel is $\ln (\omega)$ vs. $\theta$. Lower panel is $\ln (\omega)$ vs. $\ln (\theta)$, where $\theta$ is the angular separation between pairs of stars. 


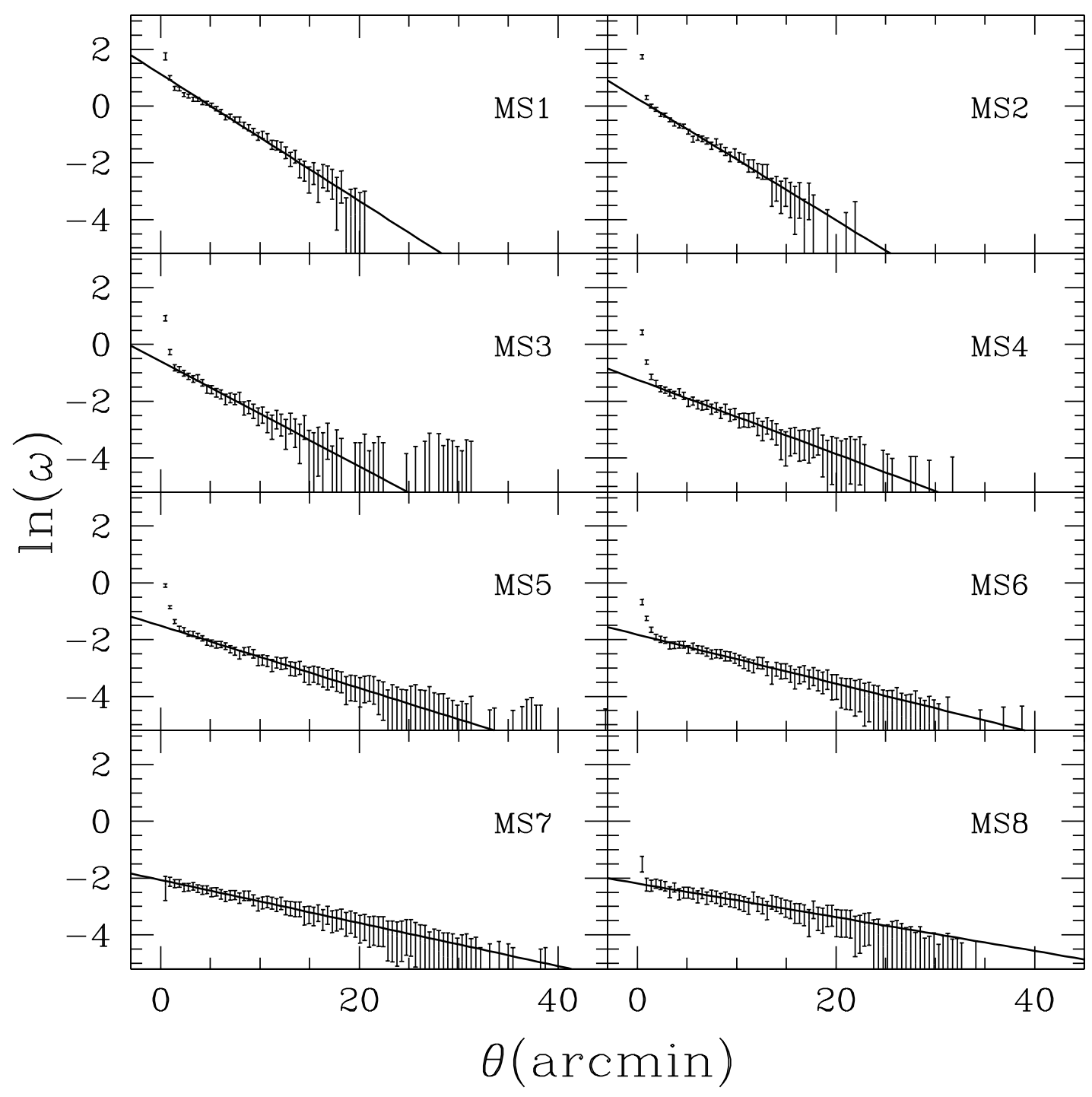

Fig. 4.- Correlation functions of the eight MS populations. Solid lines are least squares fits over the range $2<\theta<16$ arcmin for MS1, MS2 and MS3, and $2<\theta<20$ arcmin otherwise. 


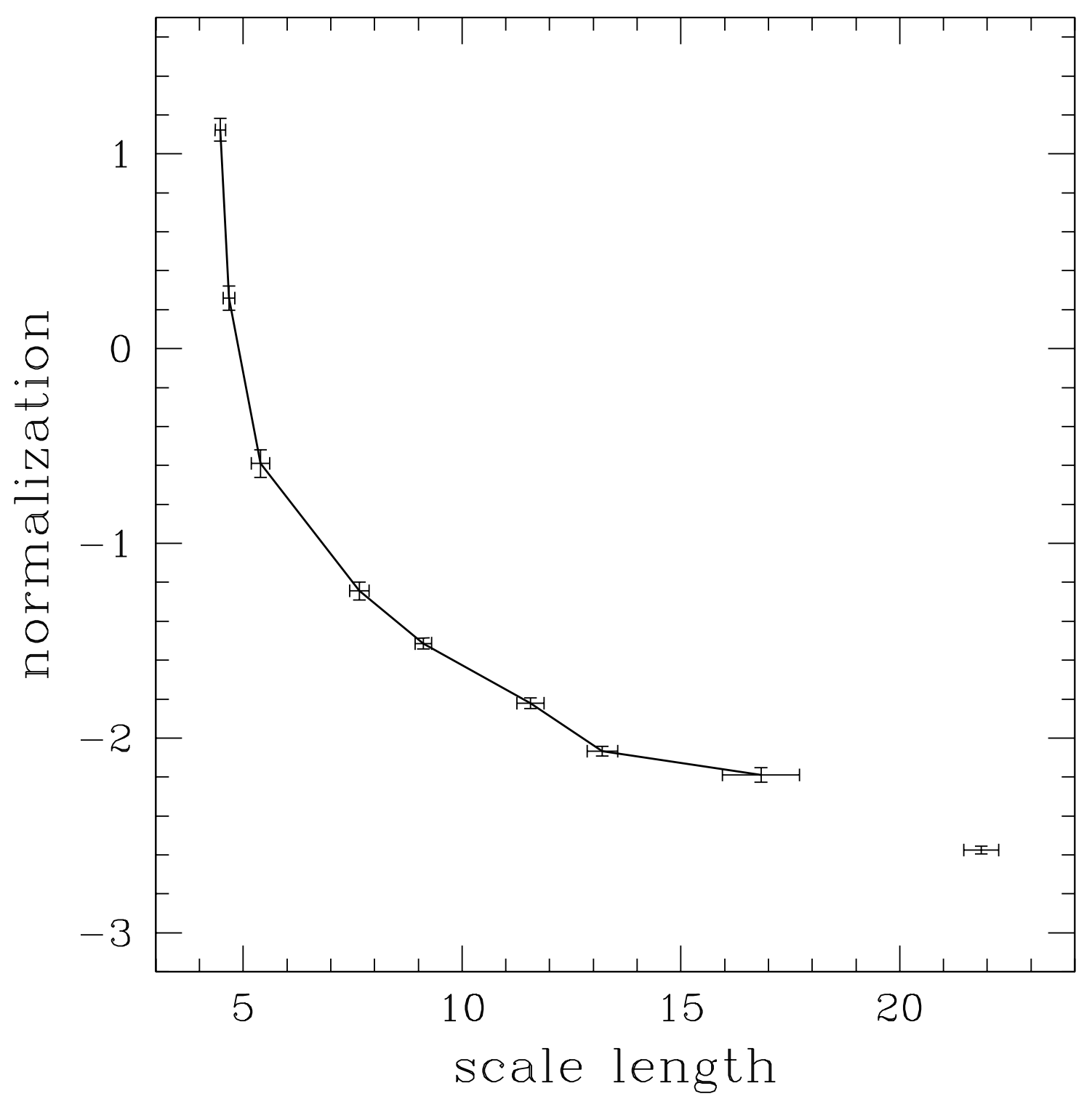

Fig. 5.- Parameterization of the best-fit exponentials to the eight MS correlation functions plotted in Figure 6 . The results for MS1 are in the upper left of the Figure and the results for MS8 are in the lower right. The unconnected point is the parameterization of the RC population's correlation function. 


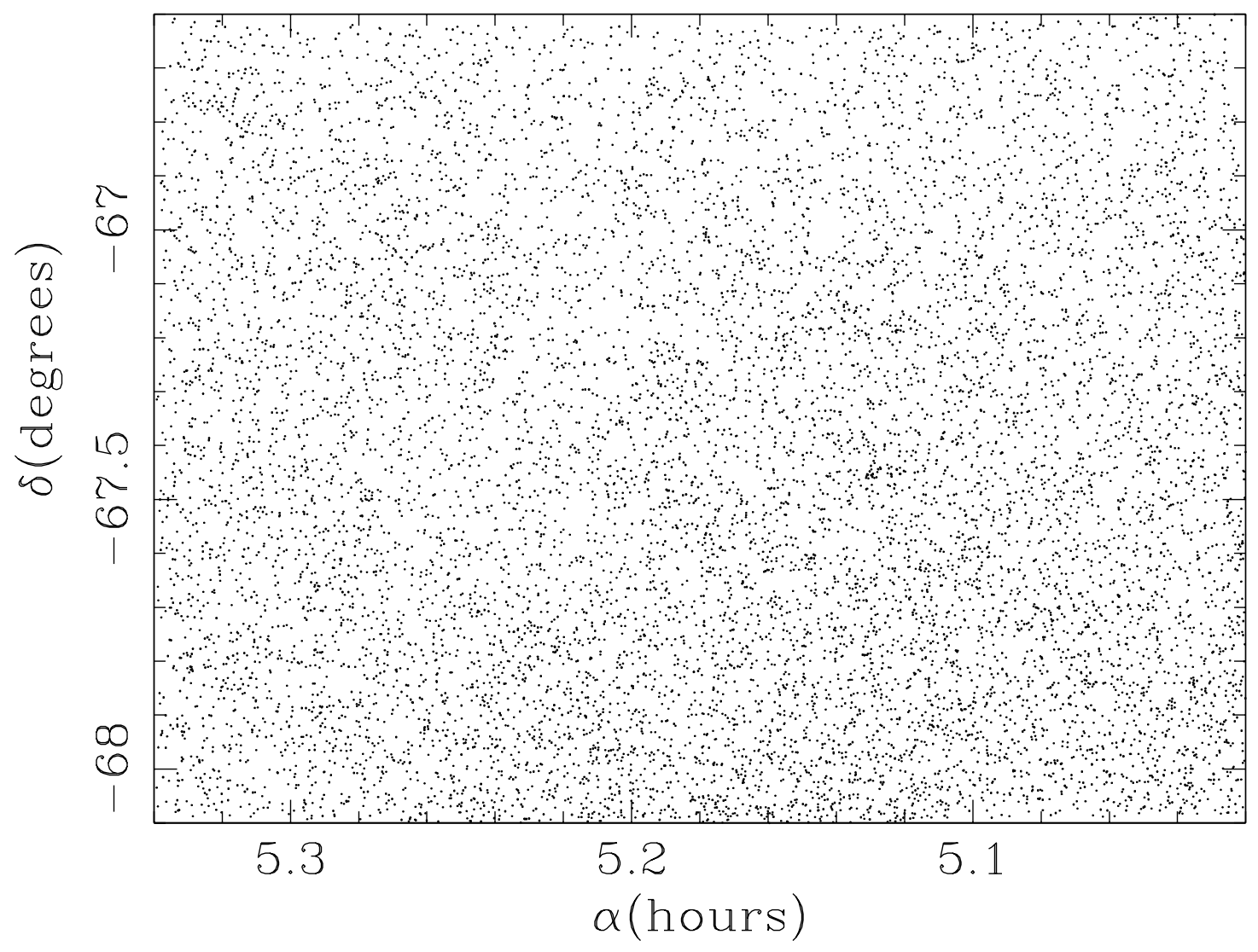

Fig. 6.- The distribution on the sky of the red clump (RC) population. For clarity, only $10 \%$ of the $\sim 100,000$ RC stars are displayed. Comparison to Figure 2 illustrates that the MS1 stars are much more clustered than the RC stars. 

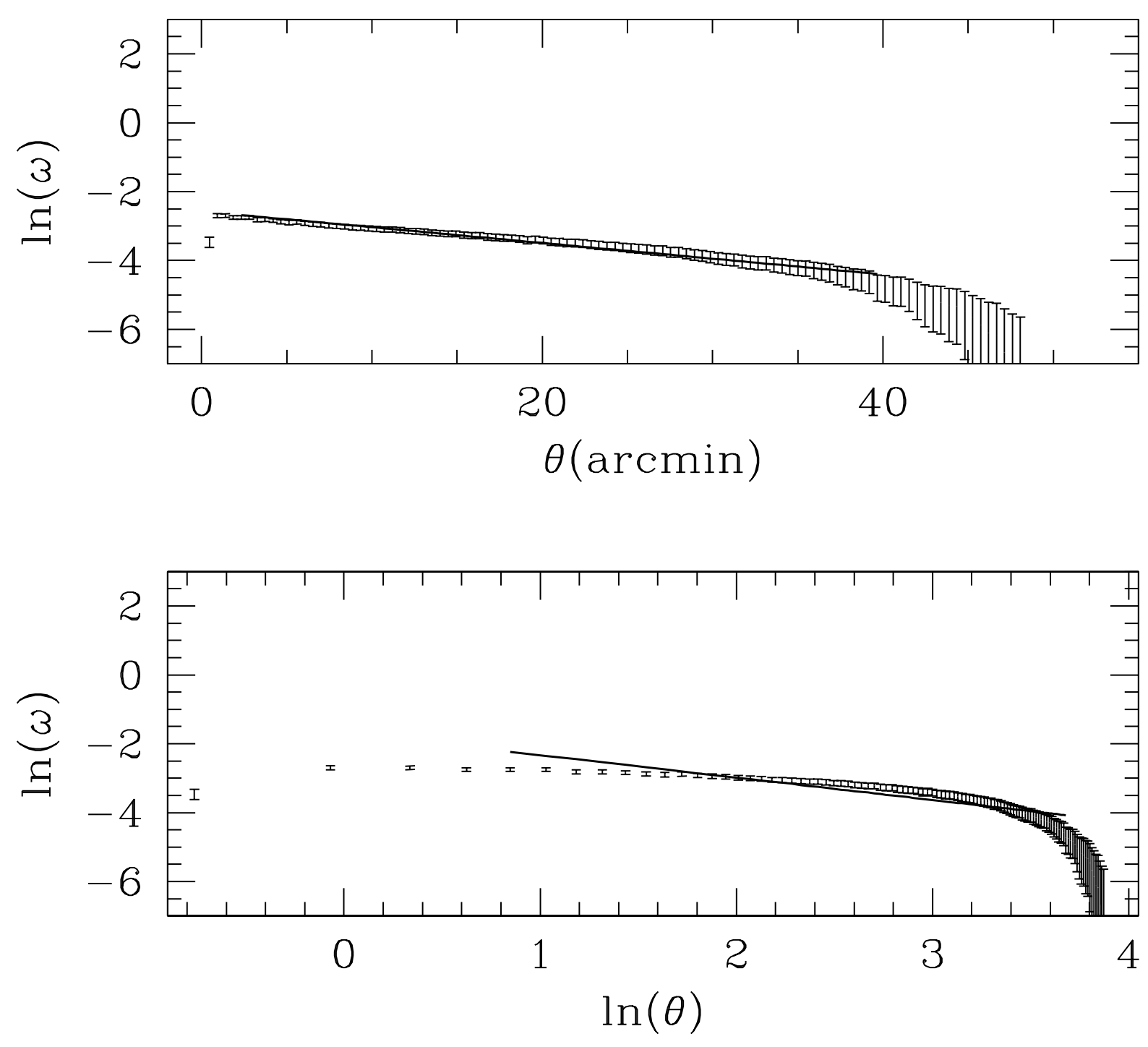

Fig. 7.- The correlation function of the RC population. 


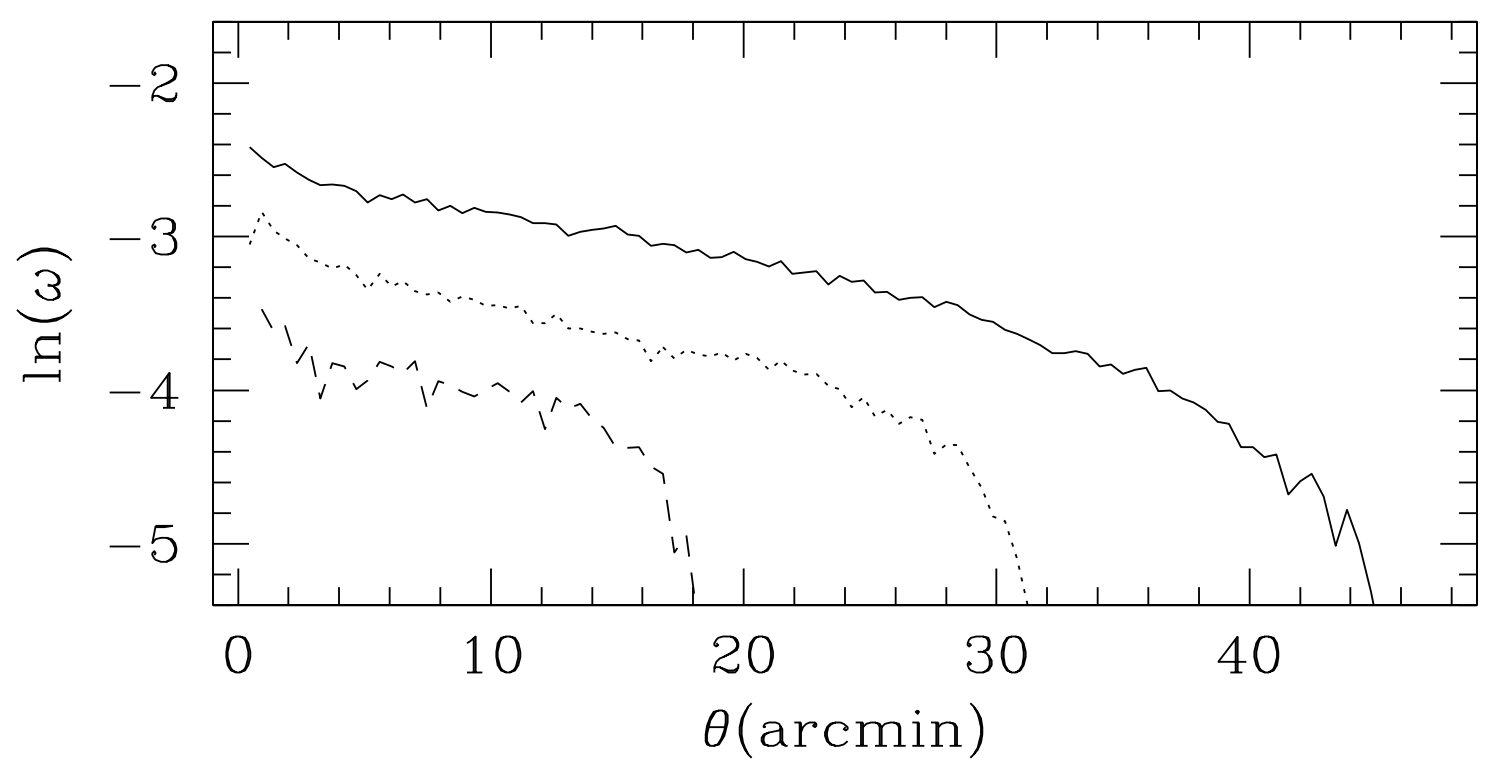

Fig. 8.- Correlation functions of three subregions of the RC population. The subregions are concentric with the original observed region, but their sizes are $1.45^{\circ} \times 1.45^{\circ}$ (solid line), $1.0^{\circ} \times 1.0^{\circ}$ (dotted line), and $0.5^{\circ} \times 0.5^{\circ}$ (dashed line). The correlation functions are offset vertically because the correlation is due to a gradient in the surface density of RC stars. The cutoff separation at which the populations become uncorrelated occurs at approximately 0.5 - 0.65 times the angular size of the observed region. 


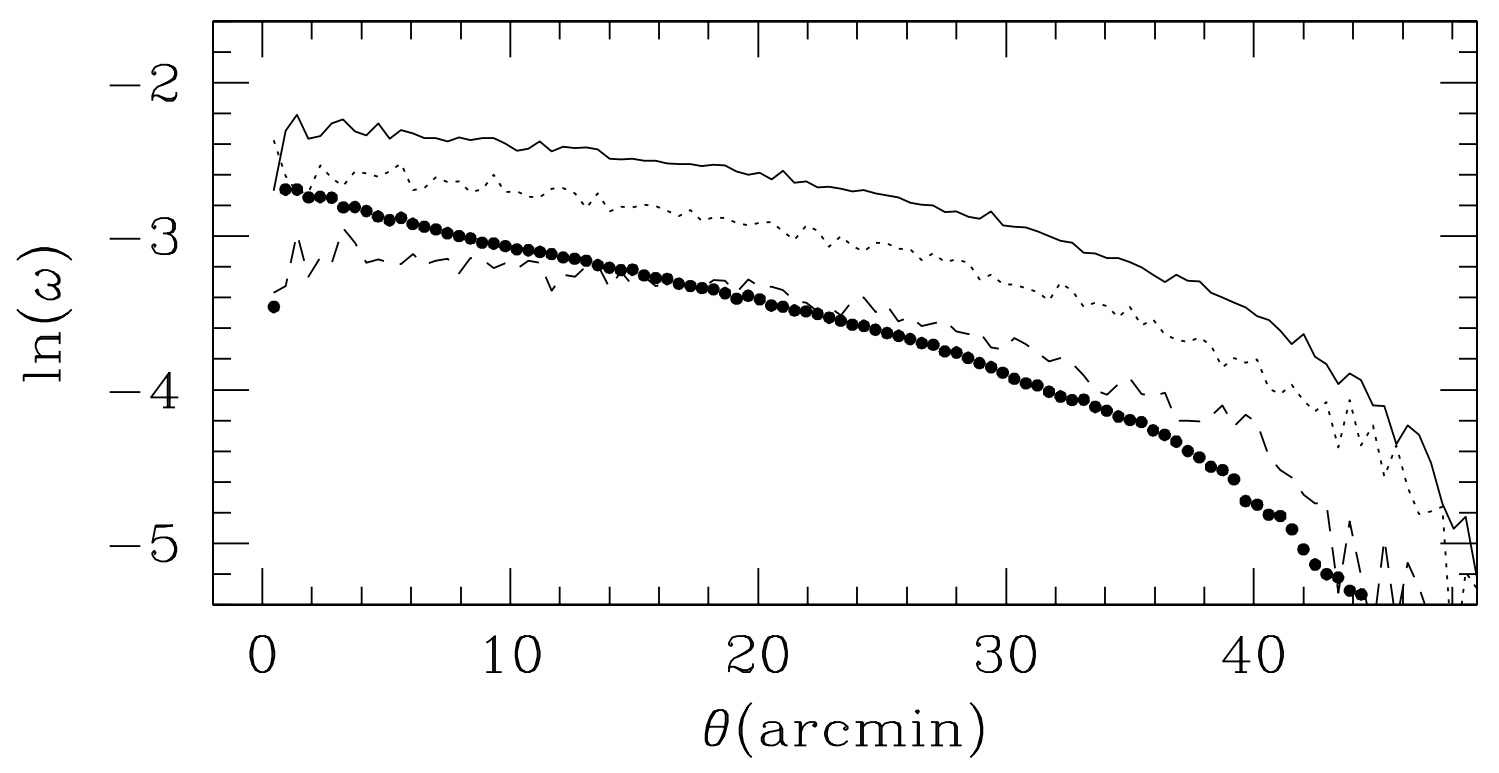

Fig. 9.- Comparison of the RC population correlation function (points) to that of an artificial exponential-disk population with a radial scale length of 101 arcminutes (solid line). We also constructed artificial populations in which the radial scale length was 115 arcminutes (dotted line) and 130 arcminutes (dashed line). 


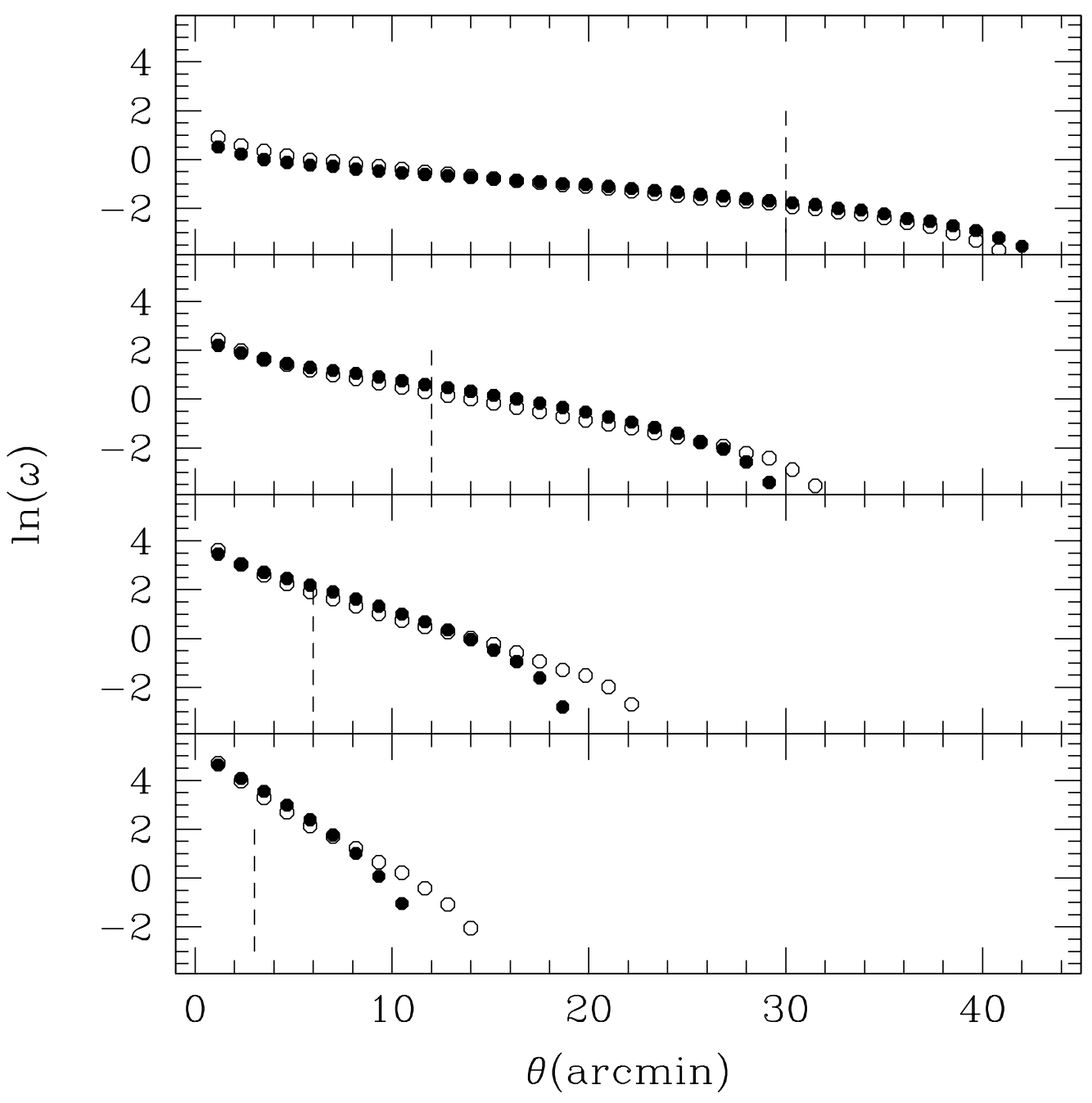

Fig. 10.- The correlation functions of eight simulations containing individual star clusters. The filled points represent results obtained using clusters with a Gaussian radial stellar density profile, the open points represent results using exponential radial profiles. Each panel contains the results using clusters with different characteristic angular size, which is indicated by the dashed vertical line. 


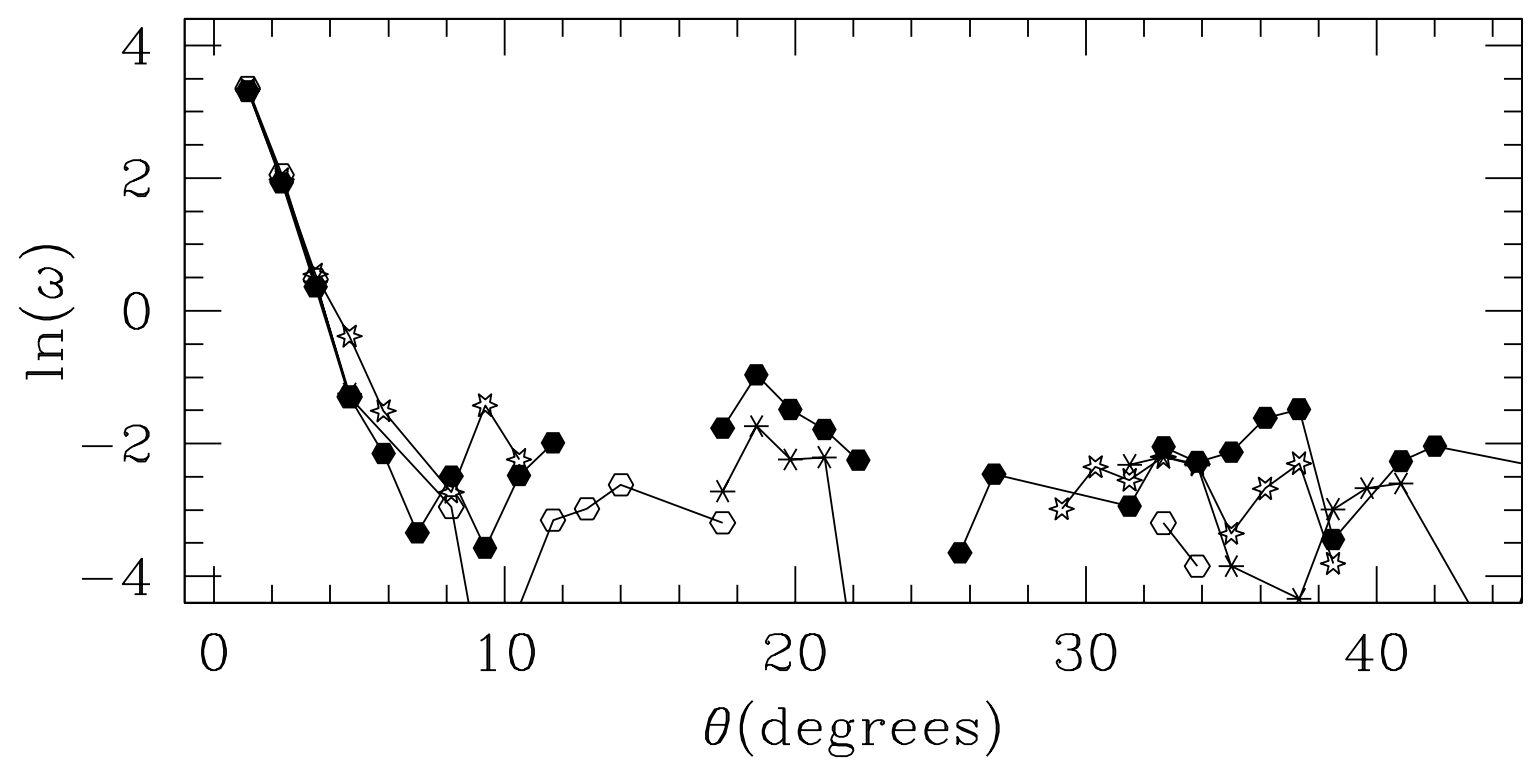

Fig. 11.- The correlation functions of four simulations containing 20 randomly distributed star clusters with exponential radial profiles and characteristic angular size of 3 arcmin. The four simulations differed only in random seed, and each is represented by a different point style. 


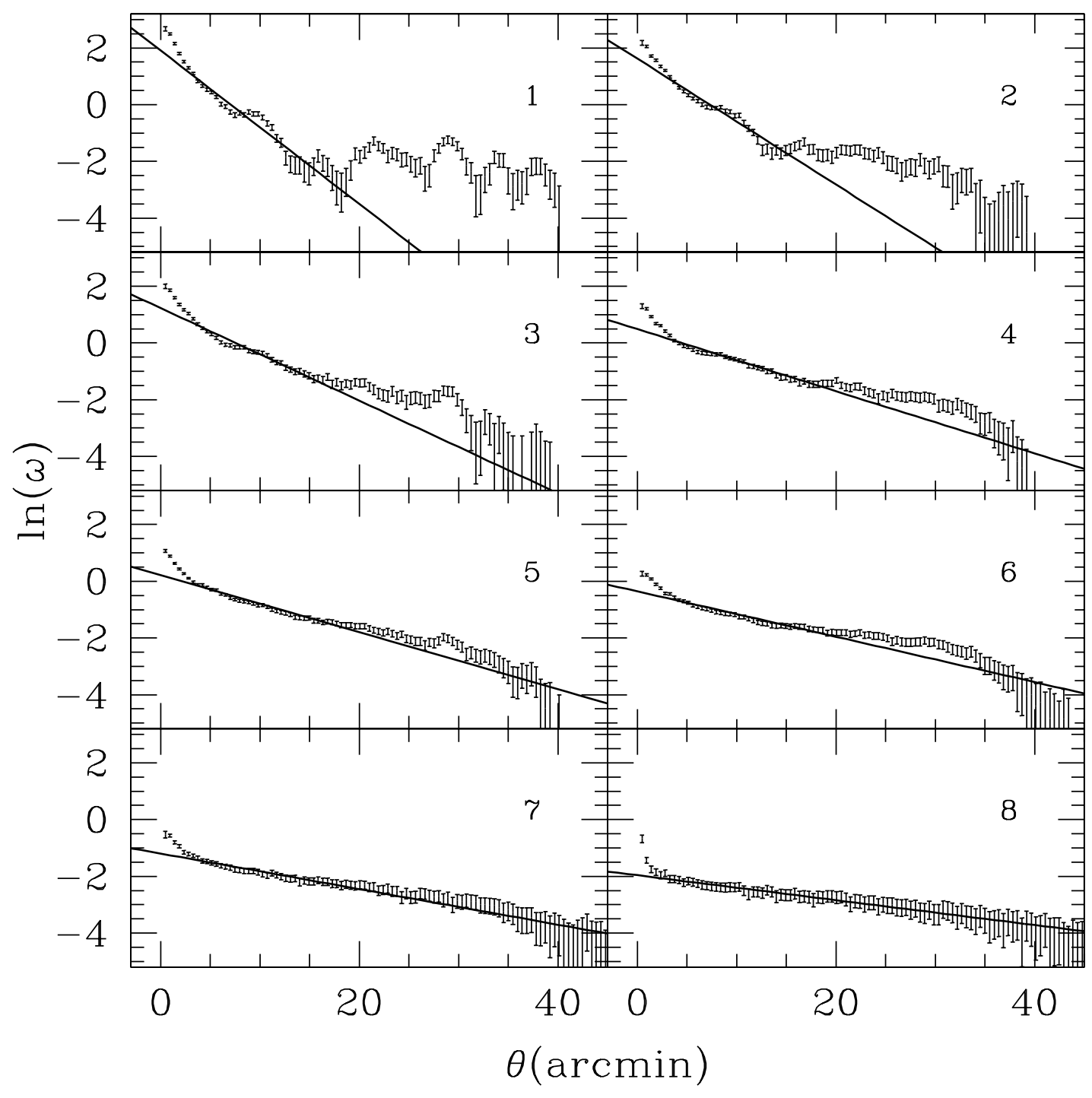

Fig. 12. - The eight MS correlation functions of our best-fitting SSPSF model. 


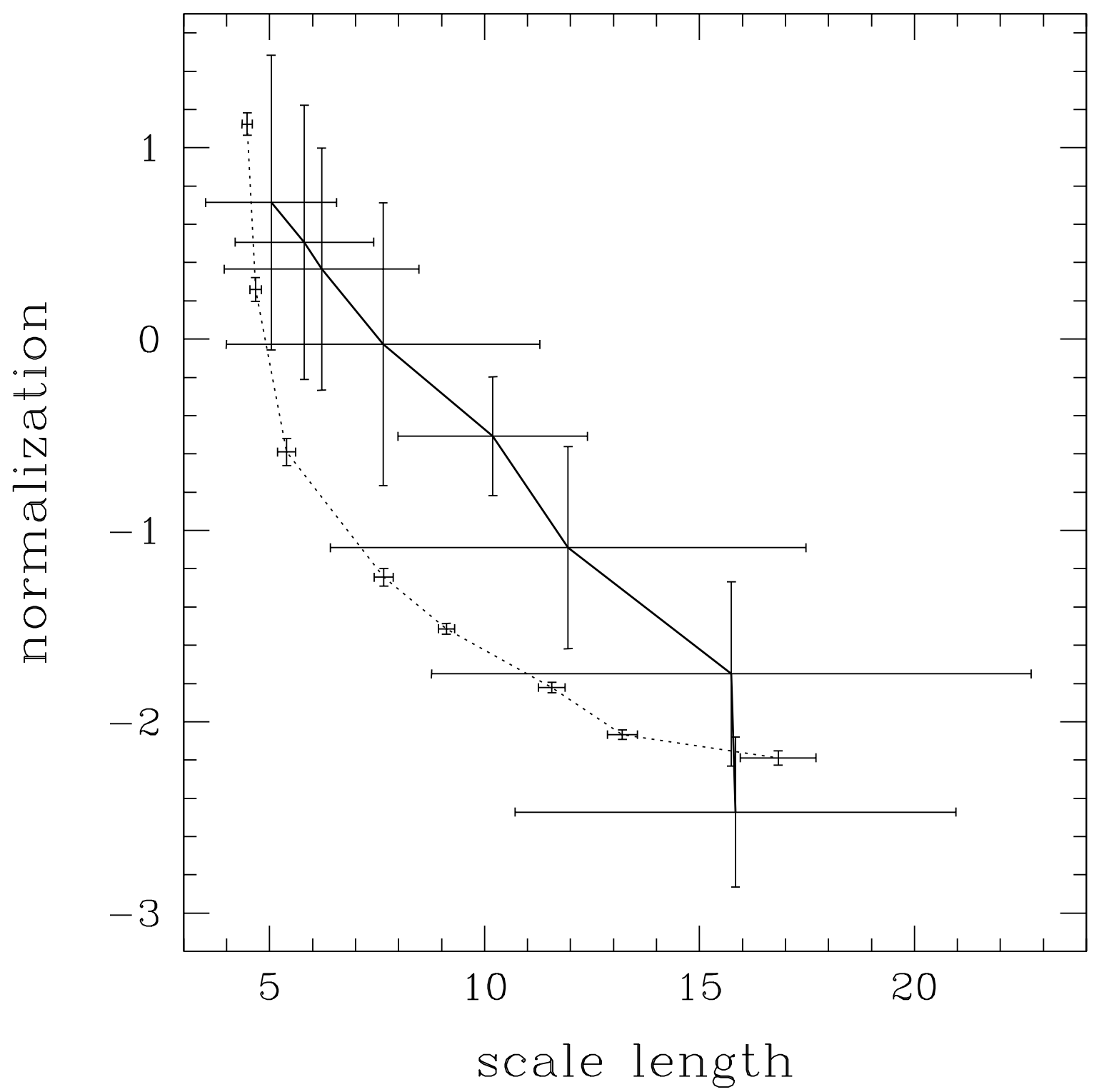

Fig. 13.- Parameterization of the best-fit models to the correlation functions of our SSPSF model (solid line). Also shown is the parameterization of the observations (dashed line) reproduced from Figure 5 for comparison. 